\title{
SNO+: Current Results and Future Prospects
}

\author{
E. J. Leming on behalf of the SNO+ collaboration* \\ University of Oxford \\ E-mail: edward.leming@physics.ox.ac.uk
}

$\mathrm{SNO}+$ is a multipurpose neutrino experiment located approximately $2 \mathrm{~km}$ underground at SNOLAB, near Sudbury, Canada. The experiment takes a phased approach, transitioning through a water phase and an ultra-pure scintillator phase before loading the liquid scintillator with $0.5 \%$ natural Tellurium, which corresponds to approximately $1330 \mathrm{~kg}$ of ${ }^{130} \mathrm{Te}$. Water phase began in May 2017 resulting in a number of fundamental physics measurements including: a limit on invisible nucleon decay [1] and a measurement of the ${ }^{8} \mathrm{Be}$ solar neutrino flux [2]. Scintillator fill began in November 2018 and Tellurium loading is planned for late 2019. During the loaded scintillator phase SNO+ will search for the neutrinoless double-beta decay of ${ }^{130} \mathrm{Te}$ with an expected effective Majorana neutrino mass sensitivity which will probe the boundary of the inverted mass hierarchy. Additionally, SNO+ aims to measure reactor antineutrino oscillations, low-energy solar neutrinos, geo-neutrinos, to be sensitive to supernova neutrinos, and to search for exotic physics.

Neutrino Oscillation Workshop (NOW2018)

9 - 16 September, 2018

Rosa Marina (Ostuni, Brindisi, Italy)

* Speaker.

${ }^{\dagger}$ A footnote may follow. 


\begin{tabular}{l|c}
\hline \hline & Liftime limits \\
\hline $\mathrm{p}$ & $2.5 \times 10^{29} \mathrm{y}$ \\
$\mathrm{n}$ & $3.6 \times 10^{29} \mathrm{y}$ \\
$\mathrm{pp}$ & $4.7 \times 10^{28} \mathrm{y}$ \\
$\mathrm{pn}$ & $2.6 \times 10^{28} \mathrm{y}$ \\
$\mathrm{nn}$ & $1.3 \times 10^{28} \mathrm{y}$ \\
\hline \hline
\end{tabular}

Table 1: Single and dinucleaon decay lifetime limits at $90 \%$ C.I. calculated as given in [1]

\section{Introduction}

$\mathrm{SNO}+$ is a large-scale liquid scintillator experiment located at a depth of 5890 meters water equivalent in the Vale's Creighton mine in Sudbury, Canada. The detector consists of a $12 \mathrm{~m}$ diameter acrylic vessel (AV) into which the detection material can be filled. Outside the AV approximately 9400 PMTs are mounted on an $18 \mathrm{~m}$ diameter geodesic stainless steel structure (PSUP) pointing inwards towards the AV. The whole experiment is placed in a cavity filled with 7000 tonnes of ultra-pure water. The ultra-pure water provides shielding against both ambient radioactive backgrounds in the cavity rock and gamma rays emanating from the PMTs entering the sensitive volume. A complete description of the detector can be found in [3].

The experiment takes a phased approach, changing the detector medium inside the AV in order to target a number of physics goals. The initial phase of the experiment employs ultra-pure water which is then replaced with ultra-pure liquid scintillator and finally loaded with Tellurium. Each phase will be used to perform both fundamental physics measurements and characterise the detector in view of setting the best possible limit on the neutrinoless double beta decay of ${ }^{130} \mathrm{Te}$.

\section{Water Phase Results}

\subsection{Invisible Nucleon Decay}

A major physics goal from water phase was the search for 'invisible' nucleon decay. Here 'invisible' refers to decays where none of the final state particles interact with the detector, for example: ${ }^{16} \mathrm{O} \rightarrow{ }^{15} \mathrm{O}^{*}+\bar{v} \bar{v} v$. The de-excitation of ${ }^{15} \mathrm{O}^{*}$ creates a gamma around 6-7 MeV with a branching ratio of approximately 0.45 . Searches for similar gamma decays have been run in mind of setting limits on both single nucleon and dinucleon decay modes [1].

A summary of the measured lifetime limits for all search channels is given in Table 1. The proton decay lifetime measurement represents a 70\% improvement on the previous limit from SNO, while pn and pp limits represent an improvement over existing limits by close to three orders of magnitude.

\section{$2.2{ }^{8}$ B Solar Neutrino Flux}

A water phase measurement of the ${ }^{8} \mathrm{~B}$ solar neutrino flux has also been made finding a value of $2.53_{-0.28}^{+0.31}$ (stat. $)_{-0.10}^{+0.13}$ (syst.) $\times 10^{6} \mathrm{~cm}^{-2} \mathrm{~s}^{-1}$, assuming no neutrino oscillations [2]. This rate is consistent with matter enhanced neutrino oscillations and measurements from other experiments. 
Due to the large rock overburden at SNOLAB the cosmic-ray muon rate within the detector volume is very low. SNO+ therefore observes remarkably low background rates at energies above $6 \mathrm{MeV}$, as shown in Figure 1. This, in conjunction with the measured systematic uncertainties, allows for an accurate measurement of the solar neutrino flux with a relatively small data set.

\section{Neutrinoless Double-Beta Decay}

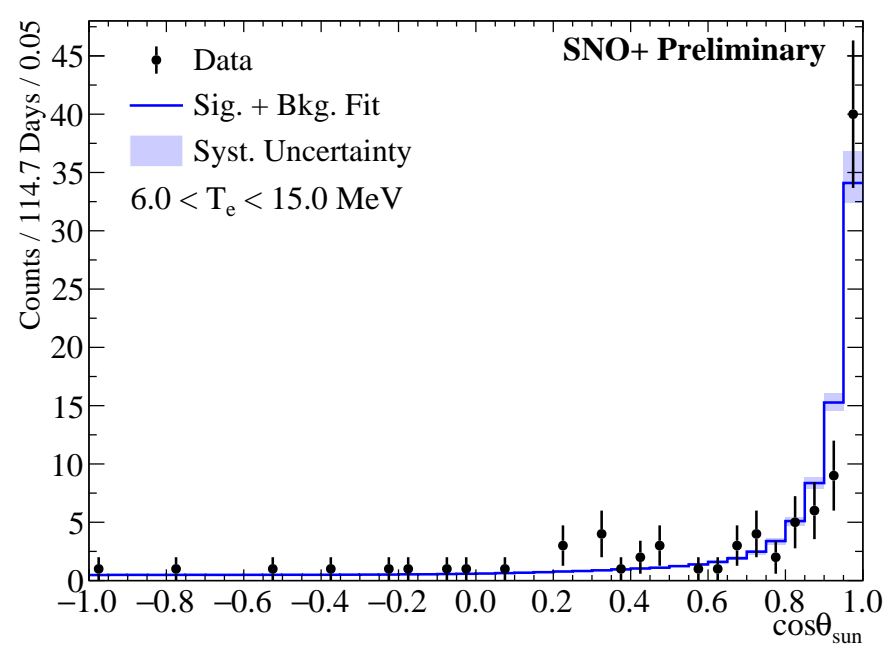

Figure 1: Distribution of event directions with respect to solar direction for events with energy in $6.0-15.0 \mathrm{MeV}$ [2].

The main physics goal of the SNO+ experiment is to search for neutrinoless double beta decay in ${ }^{130} \mathrm{Te}$. To this end, in the Tellurium phase, the liquid scintillator will initially be loaded with $0.5 \%$ of natural tellurium by mass, corresponding to about $1300 \mathrm{~kg}$ of ${ }^{130} \mathrm{Te}$.

Figure $2 \mathrm{a}$ shows the expected background in the region of interest (ROI) for this detector configuration. The ROI is centered on the ${ }^{130} \mathrm{Te} \mathrm{Q}$-value but is asymmetric $[-0.5 \sigma ;+1.5 \sigma]$ to minimise the contribution of the large 2-neutrino double-beta background. The three largest backgrounds to this measurement are predicted to be: Elastic scattering of solar neutrinos from ${ }^{8} \mathrm{~B}$; Internal uranium and thorium contaminations and external gamma rays (from PMTs, rock, etc..) respectively. As part of the water phase physics program the external gamma-ray rate has been measured and is found to be consistent with expectations [1]. After 5 years of data taking, and with these expected background and signal rates, $\mathrm{SNO}+$ aims at reaching a sensitivity on the half-life of the neutrinoless double-beta decay in ${ }^{130} \mathrm{Te}$ of $1.9 \times 10^{26}$ years, which corresponds to a limit on the effective Majorana mass in the range $41-99 \mathrm{meV}$.

The energy spectra of the aforementioned backgrounds, as well as the expected energy spectrum of the neutrinoless double-beta decay signal for an effective Majorana mass of $100 \mathrm{meV}$ after 5 years of data taking and a Te-loading of $0.5 \%$, are shown in Figure $2 b$.

\section{Conclusions}

The first physics results from the SNO+ experiment are now available on the ar $\chi$ ive. A measurement of the lifetime limits of single-nucleon and dinucleon 'invisible' decay rates has been made, setting world leading limits for multiple modes. Additionally the ${ }^{8} \mathrm{~B}$ solar neutrino flux has been measured at a rate consistent with other experiments. In producing these analyses the 'external' background rates (i.e. gamma-ray photons emanating from the PMTs) are shown to be consistent with expectations, ensuring they are well constrained for future phase measurements. 


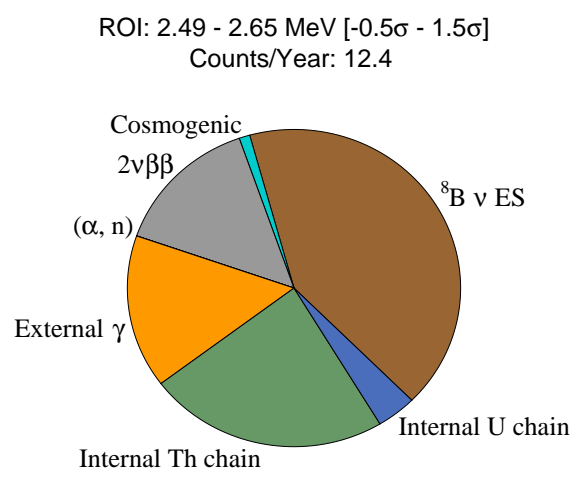

(a) Background estimation for $\mathrm{SNO}+$ tellurium phase.

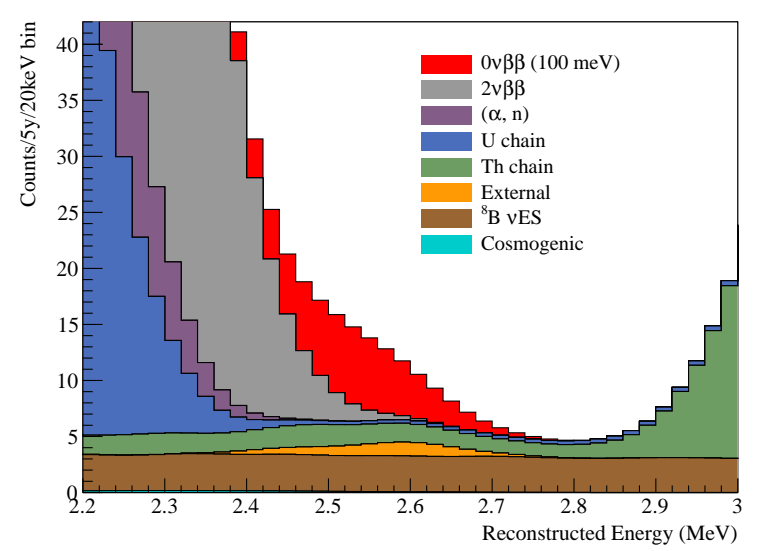

(b) Expected energy spectrum in the region of interest, showing all backgrounds and signal

Figure 2: Estimated background contributions and expected energy spectra for neutrinoless doublebeta decay in $\mathrm{SNO}+$.

The nominal loading of $\mathrm{SNO}+$ in its Tellurium phase as $0.5 \%$ by mass, corresponding to a predicted limit of the double beta half-life of $1.9 \times 10^{26}$ years. However, studies performed on higher concentrations of Tellurium show that concentrations up to $3 \%$ by mass can be reached without significant losses of light transmission in the detector. The versatility of SNO+ and its loading technique could thus allow it to increase the concentration of Tellurium in the future in order to reach limits on the neutrinoless double-beta half-life of more than $10^{27}$ years, making it possible to start probing the inverted hierarchy domain of the mass phase space.

\section{Acknowledgements}

This work is supported by STFC, ASRIP, CIFAR, CFI, DF, DOE, ERC, FCT, FedNor, NSERC, NSF, Ontario MRI, Queen's University, UC Berkeley and benefited from services provided by EGI, GridPP and Compute Canada. We thank SNOLAB and Vale for valuable support.

\section{References}

[1] M. Anderson et al. [SNO+ Collaboration], "Search for invisible modes of nucleon decay in water with the SNO+ detector," arXiv:1812.05552 [hep-ex].

[2] M. Anderson et al. [SNO+ Collaboration], "Measurement of the ${ }^{8} \mathrm{~B}$ Solar Neutrino Flux in SNO+ with Very Low Backgrounds," arXiv:1812.03355 [hep-ex].

[3] S. Andringa et al. [SNO+ Collaboration], "Current Status and Future Prospects of the SNO+ Experiment," Adv. High Energy Phys. 2016 (2016) 6194250 doi:10.1155/2016/6194250 [arXiv:1508.05759 [physics.ins-det]]. 